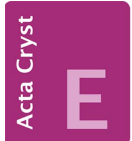

CRYSTALLOGRAPHIC COMMUNICATIONS

ISSN 2056-9890

\section{Crystal structure of oxadiargyl}

\author{
Gihaeng Kang, Jineun Kim, ${ }^{*}$ Hyunjin Park and Tae Ho \\ Kim*
}

Department of Chemistry and Research Institute of Natural Sciences, Gyeongsang, National University, Jinju 660-701, Republic of Korea. *Correspondence e-mail:

thkim@gnu.ac.kr, jekim@gnu.ac.kr

Received 5 June 2015; accepted 15 June 2015

Edited by J. Simpson, University of Otago, New Zealand

In the title compound \{systematic name: 5-tert-butyl-3-[2,4dichloro-5-(prop-2-ynyloxy)phenyl]-1,3,4-oxadiazol-2(3H)one\}, $\mathrm{C}_{15} \mathrm{H}_{14} \mathrm{Cl}_{2} \mathrm{~N}_{2} \mathrm{O}_{3}$, which is an oxadiazolone herbicide, the dihedral angle between the planes of the oxadiazolone and benzene rings is $65.84(6)^{\circ}$. In the crystal, weak intermolecular $\mathrm{Cl}$ ‥Cl [3.3600 (7) $\mathrm{A}]$ short contacts link adjacent molecules, forming chains along the $b$-axis direction. These chains are linked by $\mathrm{C}-\mathrm{H} \cdots \mathrm{O}, \mathrm{C}-\mathrm{H} \cdots \mathrm{N}$ and $\mathrm{C}-\mathrm{H} \cdots \mathrm{Cl}$ hydrogen bonds, generating a three-dimensional network. Weak $\mathrm{C}-$ $\mathrm{H} \cdots \pi$ interactions are also present.

Keywords: crystal structure; oxadiargyl; 1,3,4-oxadiazolone; herbicide; hydrogen bonding; $\mathrm{Cl} \ldots \mathrm{Cl}$ short contacts.

CCDC reference: 1406766

\section{Related literature}

For information on the herbicidal properties of the title compound, see: Saber-Tehrani et al. (2012). For a related crystal structure, see: Zhang (2006).

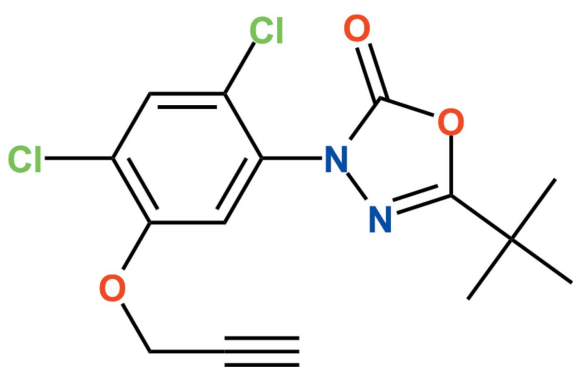

\section{Experimental}

\subsection{Crystal data}

$\mathrm{C}_{15} \mathrm{H}_{14} \mathrm{Cl}_{2} \mathrm{~N}_{2} \mathrm{O}_{3}$

$M_{r}=341.18$
Monoclinic, $P 2_{1} / c$

$a=12.9132(6) \AA$

$b=15.3893$ (7) $\AA$

$c=8.4792(4) \AA$

$\beta=107.559(1)^{\circ}$

$V=1606.52(13) \AA^{3}$

\subsection{Data collection}

Bruker APEXII CCD diffractometer

Absorption correction: multi-scan

(SADABS; Bruker, 2009)

$T_{\min }=0.878, T_{\max }=0.984$

\subsection{Refinement}

$R\left[F^{2}>2 \sigma\left(F^{2}\right)\right]=0.036$

$w R\left(F^{2}\right)=0.091$

$S=1.03$

3673 reflections

202 parameters

$\mathrm{H}$-atom parameters constrained

$\Delta \rho_{\max }=0.37 \mathrm{e}^{-3}$

$\Delta \rho_{\min }=-0.50 \mathrm{e}^{-3}$

Table 1

Hydrogen-bond geometry $\left(\AA{ }^{\circ}\right)$.

$C g 1$ and $C g 2$ are the centroids of the $\mathrm{O} 3 / \mathrm{C} 10 / \mathrm{N} 1 / \mathrm{N} 2 / \mathrm{C} 11$ and $\mathrm{C} 4-\mathrm{C} 9$ rings, respectively.

\begin{tabular}{lllll}
\hline$D-\mathrm{H} \cdots A$ & $D-\mathrm{H}$ & $\mathrm{H} \cdots A$ & $D \cdots A$ & $D-\mathrm{H} \cdots A$ \\
\hline $\mathrm{C} 1-\mathrm{H} 1 \cdots \mathrm{O}^{\mathrm{i}}$ & 0.95 & 2.47 & $3.401(3)$ & 168 \\
$\mathrm{C} 13-\mathrm{H} 13 B \cdots \mathrm{O}^{\mathrm{ii}}$ & 0.98 & 2.51 & $3.429(2)$ & 155 \\
$\mathrm{C} 3-\mathrm{H} 3 B \cdots \mathrm{N}{ }^{2 i i}$ & 0.99 & 2.64 & $3.607(2)$ & 166 \\
$\mathrm{C} 13-\mathrm{H} 13 A \cdots \mathrm{Cl} 1^{\mathrm{i}}$ & 0.98 & 2.85 & $3.811(2)$ & 168 \\
$\mathrm{C} 14-\mathrm{H} 14 \mathrm{~b} \cdots C 2^{\mathrm{ii}}$ & 0.98 & 2.99 & $3.396(2)$ & 106 \\
$\mathrm{C} 15-\mathrm{H} 15 \cdots C g 1^{\mathrm{v}}$ & 0.98 & 2.80 & $3.497(2)$ & 129 \\
\hline
\end{tabular}

Symmetry codes: (i) $-x,-y+2,-z$; (ii) $x, y, z+1$; (iii) $x,-y+\frac{3}{2}, z-\frac{1}{2}$; (iv) $-x+1,-y+2,-z+1 ;(\mathrm{v})-x,-y+2,-z+2$.

Data collection: APEX2 (Bruker 2009); cell refinement: SAINT (Bruker 2009); data reduction: $S A I N T$; $\operatorname{program}(\mathrm{s})$ used to solve structure: SHELXS97 (Sheldrick 2008); program(s) used to refine structure: SHELXL2013 (Sheldrick, 2015); molecular graphics: DIAMOND (Brandenburg, 2010); software used to prepare material for publication: SHELXTL (Sheldrick 2008).

\section{Acknowledgements}

This research was supported by the Basic Science Research Program through the National Research Foundation of Korea (NRF) funded by the Ministry of Education, Science and Technology (No. 2014R1A1A4A01009105).

Supporting information for this paper is available from the IUCr electronic archives (Reference: SJ5464).

\section{References}

Brandenburg, K. (2010). DIAMOND. Crystal Impact GbR, Bonn, Germany. Bruker (2009). APEX2, SAINT and SADABS. Bruker AXS Inc., Madison, Wisconsin, USA.

Saber-Tehrani, M., Pourhabib, A., Husain, S. W. \& Arvand, M. (2012). Electroanalysis, 24, 2395-2404.

Sheldrick, G. M. (2008). Acta Cryst. A64, 112-122.

Sheldrick, G. M. (2015). Acta Cryst. C71, 3-8.

Zhang, G.-L. (2006). Acta Cryst. E62, o1556-o1557. 


\section{supporting information}

Acta Cryst. (2015). E71, o494 [doi:10.1107/S2056989015011524]

\section{Crystal structure of oxadiargyl}

\section{Gihaeng Kang, Jineun Kim, Hyunjin Park and Tae Ho Kim}

\section{S1. Comment}

Oxadiargyl [systematic name: 5-tert-butyl-3-[2,4-dichloro-5-(prop-2- ynyloxy)phenyl]-1,3,4-oxadiazol-2(3H)-one] is an oxadiazolone herbicide that has been developed for the control of annual grasses, sedges and broad-leaf weeds in rice fields (Saber-Tehrani et al., 2012). However, until now its crystal structure has not been reported. In the title compound (Fig. 1), the dihedral angle between the planes of the oxadiazolone and benzene rings is $65.84(6)^{\circ}$. All bond lengths and bond angles are normal and comparable to those observed in the crystal structure of a similar compound (Zhang 2006). In the crystal structure (Fig. 2), weak intermolecular $\mathrm{Cl}^{\cdots} \cdots \mathrm{Cl} 2^{\mathrm{v}}[3.3600$ (7) $\AA$ ] short contacts link adjacent molecules, forming one-dimensional chains along the $b$-axis. The chains are linked by $\mathrm{C}-\mathrm{H} \cdots \mathrm{O}, \mathrm{C}-\mathrm{H} \cdots \mathrm{N}$, and $\mathrm{C}-\mathrm{H} \cdots \mathrm{Cl}$ hydrogen bonds (Table 1), resulting in a three-dimensional architecture. In addition, weak $\mathrm{C}-\mathrm{H} \cdots \pi$ interactions involving the $\mathrm{C} 14$ and $\mathrm{C} 15$ methyl groups and the oxadiazolone and benzene rings are also found, Table 1.

\section{S2. Experimental}

The title compound was purchased from the Dr. Ehrenstorfer $\mathrm{GmbH}$ Company. Slow evaporation of a solution in $\mathrm{CH}_{3} \mathrm{CN}$ gave single crystals suitable for X-ray analysis.

\section{S3. Refinement}

All H-atoms were positioned geometrically and refined using a riding model with $\mathrm{d}(\mathrm{C}-\mathrm{H})=0.98 \AA, U_{\text {iso }}=1.2 U_{\text {eq }}(\mathrm{C})$ for the methyl groups, $\mathrm{d}(\mathrm{C}-\mathrm{H})=0.99 \AA, U_{\text {iso }}=1.2 U_{\text {eq }}(\mathrm{C})$ for the methylene $\mathrm{C}-\mathrm{H}$ and $\mathrm{d}(\mathrm{C}-\mathrm{H})=0.95 \AA, U_{\text {iso }}=1.2 U_{\text {eq }}(\mathrm{C})$ for aromatic and alkyne $\mathrm{C}-\mathrm{H}$. 


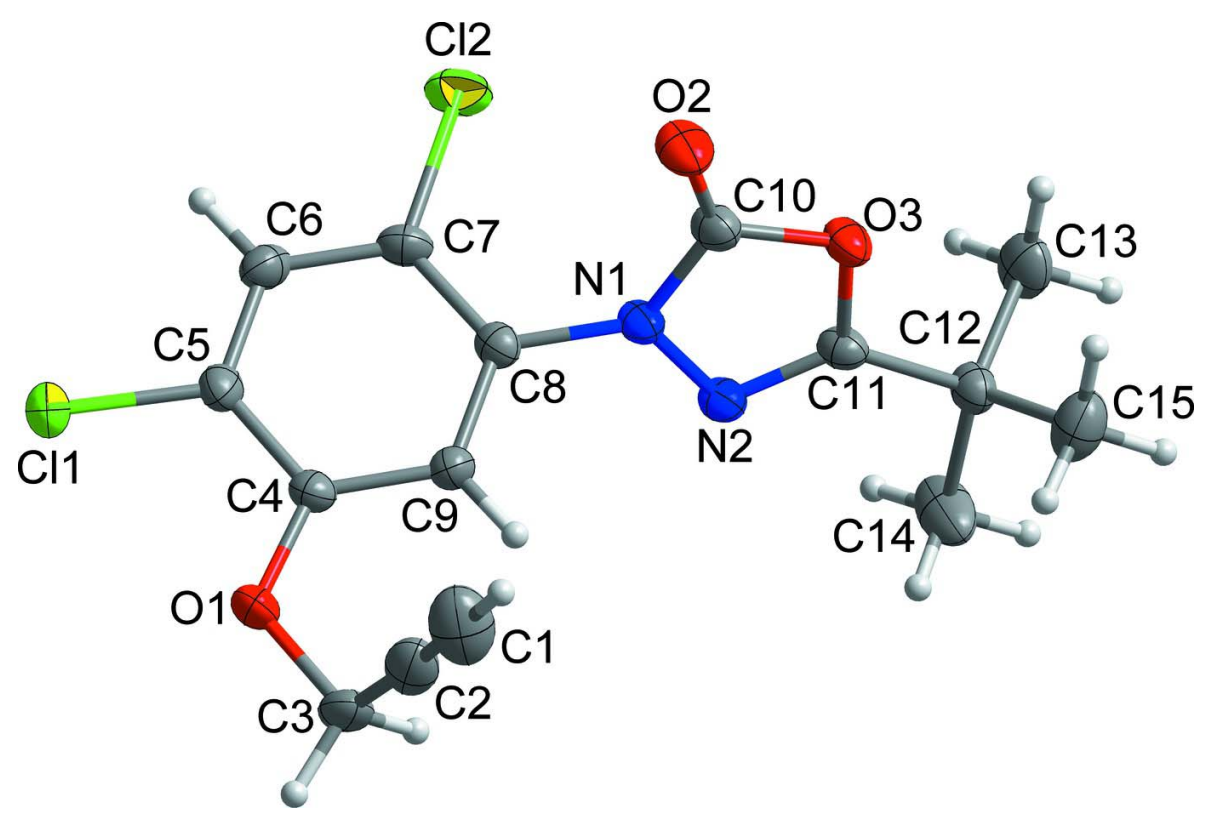

\section{Figure 1}

The asymmetric unit of the title compound with the atom-numbering scheme. Displacement ellipsoids are drawn at the $50 \%$ probability level. $\mathrm{H}$ atoms are shown as small spheres of arbitrary radius.

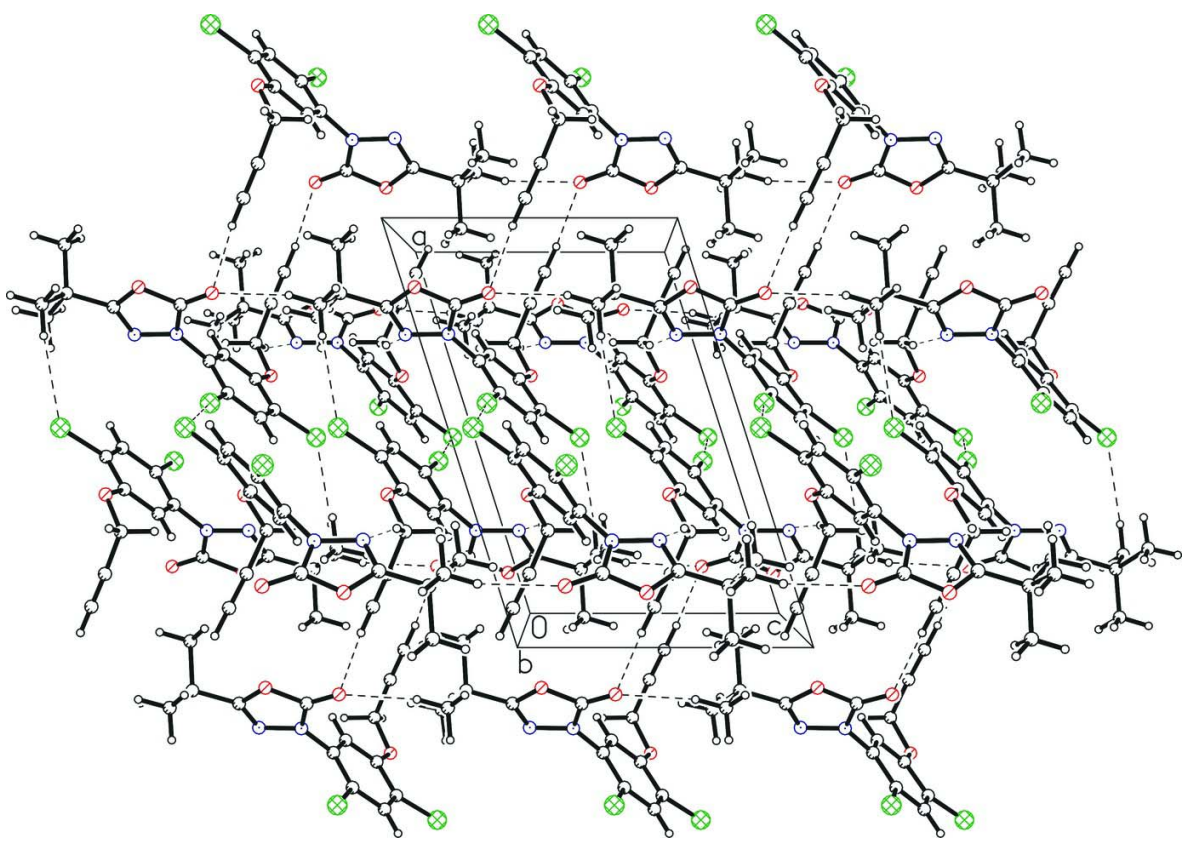

Figure 2

Crystal packing viewed along the $b$ axis. The $\mathrm{C}-\mathrm{H} \cdots \mathrm{O}$ and $\mathrm{C}-\mathrm{H} \cdots \mathrm{N}, \mathrm{C}-\mathrm{H} \cdots \mathrm{Cl}$ hydrogen bonds and short $\mathrm{Cl} \cdots \mathrm{Cl}$ contacts are shown as dashed lines. 


\section{5-tert-Butyl-3-[2,4-dichloro-5-(prop-2-ynyloxy)phenyl]-1,3,4-oxadiazol-2(3H)-one}

Crystal data

$\mathrm{C}_{15} \mathrm{H}_{14} \mathrm{Cl}_{2} \mathrm{~N}_{2} \mathrm{O}_{3}$

$M_{r}=341.18$

Monoclinic, $P 2_{1} / c$

$a=12.9132(6) \AA$

$b=15.3893(7) \AA$

$c=8.4792(4) \AA$

$\beta=107.559(1)^{\circ}$

$V=1606.52(13) \AA^{3}$

$Z=4$

Data collection

Bruker APEXII CCD

diffractometer

$\varphi$ and $\omega$ scans

Absorption correction: multi-scan

(SADABS; Bruker, 2009)

$T_{\min }=0.878, T_{\max }=0.984$

14639 measured reflections

Refinement

Refinement on $F^{2}$

Least-squares matrix: full

$R\left[F^{2}>2 \sigma\left(F^{2}\right)\right]=0.036$

$w R\left(F^{2}\right)=0.091$

$S=1.03$

3673 reflections

202 parameters

0 restraints
$F(000)=704$

$D_{\mathrm{x}}=1.411 \mathrm{Mg} \mathrm{m}^{-3}$

Mo $K \alpha$ radiation, $\lambda=0.71073 \AA$

Cell parameters from 5206 reflections

$\theta=2.7-27.4^{\circ}$

$\mu=0.42 \mathrm{~mm}^{-1}$

$T=173 \mathrm{~K}$

Block, colourless

$0.32 \times 0.14 \times 0.04 \mathrm{~mm}$

3673 independent reflections

3126 reflections with $I>2 \sigma(I)$

$R_{\text {int }}=0.030$

$\theta_{\max }=27.5^{\circ}, \theta_{\min }=2.1^{\circ}$

$h=-16 \rightarrow 15$

$k=-20 \rightarrow 19$

$l=-8 \rightarrow 11$

Hydrogen site location: inferred from neighbouring sites

$\mathrm{H}$-atom parameters constrained

$w=1 /\left[\sigma^{2}\left(F_{\mathrm{o}}^{2}\right)+(0.0373 P)^{2}+0.7866 P\right]$

where $P=\left(F_{\mathrm{o}}{ }^{2}+2 F_{\mathrm{c}}{ }^{2}\right) / 3$

$(\Delta / \sigma)_{\max }=0.001$

$\Delta \rho_{\max }=0.37 \mathrm{e}^{-3}$

$\Delta \rho_{\min }=-0.50$ e $\AA^{-3}$

Special details

Geometry. All e.s.d.'s (except the e.s.d. in the dihedral angle between two 1.s. planes) are estimated using the full covariance matrix. The cell e.s.d.'s are taken into account individually in the estimation of e.s.d.'s in distances, angles and torsion angles; correlations between e.s.d.'s in cell parameters are only used when they are defined by crystal symmetry. An approximate (isotropic) treatment of cell e.s.d.'s is used for estimating e.s.d.'s involving 1.s. planes.

Fractional atomic coordinates and isotropic or equivalent isotropic displacement parameters $\left(\hat{A}^{2}\right)$

\begin{tabular}{lllll}
\hline & $x$ & $y$ & $z$ & $U_{\text {iso }} * / U_{\text {eq }}$ \\
\hline C11 & $0.51241(4)$ & $0.84758(3)$ & $0.07299(7)$ & $0.04172(14)$ \\
C12 & $0.42630(4)$ & $1.13566(3)$ & $0.36463(6)$ & $0.03708(13)$ \\
O1 & $0.34909(9)$ & $0.76748(7)$ & $0.18615(15)$ & $0.0285(3)$ \\
O2 & $0.15195(11)$ & $1.12537(8)$ & $0.23434(14)$ & $0.0353(3)$ \\
O3 & $0.13661(9)$ & $1.11525(7)$ & $0.49542(13)$ & $0.0263(3)$ \\
N1 & $0.24959(11)$ & $1.02745(8)$ & $0.43361(16)$ & $0.0234(3)$ \\
N2 & $0.25036(11)$ & $1.00602(8)$ & $0.59469(15)$ & $0.0231(3)$ \\
C1 & $0.07451(17)$ & $0.77324(15)$ & $0.0073(3)$ & $0.0496(5)$ \\
H1 & 0.0087 & 0.7937 & -0.0683 & $0.060^{*}$ \\
C2 & $0.15618(15)$ & $0.74788(11)$ & $0.1011(2)$ & $0.0357(4)$ \\
C3 & $0.26062(15)$ & $0.71962(10)$ & $0.2137(2)$ & $0.0317(4)$
\end{tabular}




$\begin{array}{lllll}\text { H3A } & 0.2606 & 0.7281 & 0.3295 & 0.038^{*} \\ \text { H3B } & 0.2707 & 0.6569 & 0.1971 & 0.038^{*} \\ \text { C4 } & 0.35972(12) & 0.85268(9) & 0.23138(19) & 0.0215(3) \\ \text { C5 } & 0.44020(13) & 0.89889(10) & 0.1882(2) & 0.0249(3) \\ \text { C6 } & 0.46160(13) & 0.98461(10) & 0.2306(2) & 0.0264(3) \\ \text { H6 } & 0.5184 & 1.0142 & 0.2031 & 0.032^{*} \\ \text { C7 } & 0.39940(13) & 1.02756(10) & 0.3143(2) & 0.0249(3) \\ \text { C8 } & 0.31715(12) & 0.98351(10) & 0.35432(18) & 0.0218(3) \\ \text { C9 } & 0.29737(12) & 0.89626(10) & 0.31370(18) & 0.0217(3) \\ \text { H9 } & 0.2411 & 0.8665 & 0.3423 & 0.026^{*} \\ \text { C10 } & 0.17873(14) & 1.09254(10) & 0.3692(2) & 0.0259(3) \\ \text { C11 } & 0.18252(13) & 1.05977(10) & 0.62444(19) & 0.0226(3) \\ \text { C12 } & 0.14869(13) & 1.06873(10) & 0.7774(2) & 0.0262(3) \\ \text { C13 } & 0.18132(16) & 1.15945(12) & 0.8505(2) & 0.0381(4) \\ \text { H13A } & 0.2602 & 1.1664 & 0.8776 & 0.057^{*} \\ \text { H13B } & 0.1597 & 1.1663 & 0.9511 & 0.057^{*} \\ \text { H13C } & 0.1449 & 1.2036 & 0.7695 & 0.057^{*} \\ \text { C14 } & 0.2053(2) & 0.99878(14) & 0.8999(2) & 0.0506(6) \\ \text { H14A } & 0.1804 & 0.9414 & 0.8539 & 0.076^{*} \\ \text { H14B } & 0.1880 & 1.0067 & 1.0039 & 0.076^{*} \\ \text { H14C } & 0.2840 & 1.0032 & 0.9209 & 0.076^{*} \\ \text { C15 } & 0.02549(16) & 1.05801(14) & 0.7315(3) & 0.0433(5) \\ \text { H15A } & -0.0100 & 1.1030 & 0.6516 & 0.065^{*} \\ \text { H15B } & 0.0025 & 1.0636 & 0.8311 & 0.065^{*} \\ \text { H15C } & 0.0049 & 1.0005 & 0.6822 & 0.065^{*}\end{array}$

Atomic displacement parameters $\left(\AA^{2}\right)$

\begin{tabular}{lllllll}
\hline & $U^{11}$ & $U^{22}$ & $U^{33}$ & $U^{12}$ & $U^{13}$ & $U^{23}$ \\
\hline C11 & $0.0476(3)$ & $0.0308(2)$ & $0.0615(3)$ & $0.00758(19)$ & $0.0387(2)$ & $0.0042(2)$ \\
C12 & $0.0460(3)$ & $0.0210(2)$ & $0.0434(3)$ & $-0.00999(18)$ & $0.0122(2)$ & $-0.00547(17)$ \\
O1 & $0.0335(6)$ & $0.0178(5)$ & $0.0382(7)$ & $0.0008(5)$ & $0.0168(5)$ & $-0.0026(5)$ \\
O2 & $0.0489(8)$ & $0.0344(7)$ & $0.0234(6)$ & $0.0135(6)$ & $0.0120(5)$ & $0.0092(5)$ \\
O3 & $0.0342(6)$ & $0.0235(6)$ & $0.0217(6)$ & $0.0083(5)$ & $0.0090(5)$ & $0.0022(4)$ \\
N1 & $0.0316(7)$ & $0.0208(6)$ & $0.0188(6)$ & $0.0047(5)$ & $0.0090(5)$ & $0.0025(5)$ \\
N2 & $0.0299(7)$ & $0.0211(6)$ & $0.0181(6)$ & $0.0018(5)$ & $0.0071(5)$ & $0.0007(5)$ \\
C1 & $0.0382(11)$ & $0.0554(13)$ & $0.0528(13)$ & $-0.0060(10)$ & $0.0100(10)$ & $-0.0193(11)$ \\
C2 & $0.0393(10)$ & $0.0300(9)$ & $0.0424(11)$ & $-0.0101(8)$ & $0.0195(9)$ & $-0.0130(8)$ \\
C3 & $0.0430(10)$ & $0.0182(7)$ & $0.0392(10)$ & $-0.0064(7)$ & $0.0206(8)$ & $-0.0025(7)$ \\
C4 & $0.0243(8)$ & $0.0176(7)$ & $0.0218(8)$ & $0.0016(6)$ & $0.0057(6)$ & $0.0016(6)$ \\
C5 & $0.0241(8)$ & $0.0255(8)$ & $0.0268(8)$ & $0.0058(6)$ & $0.0102(6)$ & $0.0044(6)$ \\
C6 & $0.0235(8)$ & $0.0258(8)$ & $0.0307(9)$ & $-0.0018(6)$ & $0.0093(6)$ & $0.0050(7)$ \\
C7 & $0.0284(8)$ & $0.0184(7)$ & $0.0253(8)$ & $-0.0036(6)$ & $0.0042(6)$ & $-0.0004(6)$ \\
C8 & $0.0249(8)$ & $0.0216(7)$ & $0.0185(7)$ & $0.0024(6)$ & $0.0059(6)$ & $0.0001(6)$ \\
C9 & $0.0227(7)$ & $0.0204(7)$ & $0.0226(8)$ & $-0.0010(6)$ & $0.0079(6)$ & $0.0018(6)$ \\
C10 & $0.0327(9)$ & $0.0220(7)$ & $0.0232(8)$ & $0.0018(7)$ & $0.0088(7)$ & $-0.0007(6)$ \\
C11 & $0.0277(8)$ & $0.0193(7)$ & $0.0189(7)$ & $0.0008(6)$ & $0.0043(6)$ & $0.0008(6)$ \\
C12 & $0.0313(8)$ & $0.0263(8)$ & $0.0228(8)$ & $0.0048(7)$ & $0.0106(7)$ & $0.0001(6)$ \\
& & & & & &
\end{tabular}




\begin{tabular}{lllllll}
$\mathrm{C} 13$ & $0.0452(11)$ & $0.0388(10)$ & $0.0341(10)$ & $-0.0050(8)$ & $0.0178(8)$ & $-0.0130(8)$ \\
$\mathrm{C} 14$ & $0.0790(16)$ & $0.0499(12)$ & $0.0287(10)$ & $0.0289(11)$ & $0.0250(10)$ & $0.0130(9)$ \\
$\mathrm{C} 15$ & $0.0384(10)$ & $0.0552(12)$ & $0.0413(11)$ & $-0.0089(9)$ & $0.0196(9)$ & $-0.0069(9)$ \\
\hline
\end{tabular}

Geometric parameters $\left(\stackrel{A}{\circ}{ }^{\circ}\right)$

\begin{tabular}{|c|c|c|c|}
\hline $\mathrm{Cl1}-\mathrm{C} 5$ & $1.7310(16)$ & $\mathrm{C} 6-\mathrm{C} 7$ & $1.389(2)$ \\
\hline $\mathrm{Cl} 2-\mathrm{C} 7$ & $1.7266(16)$ & $\mathrm{C} 6-\mathrm{H} 6$ & 0.9500 \\
\hline $\mathrm{O} 1-\mathrm{C} 4$ & $1.3613(18)$ & $\mathrm{C} 7-\mathrm{C} 8$ & $1.386(2)$ \\
\hline $\mathrm{O} 1-\mathrm{C} 3$ & $1.4365(19)$ & $\mathrm{C} 8-\mathrm{C} 9$ & $1.391(2)$ \\
\hline $\mathrm{O} 2-\mathrm{C} 10$ & $1.2017(19)$ & C9- $\mathrm{H} 9$ & 0.9500 \\
\hline $\mathrm{O} 3-\mathrm{C} 11$ & $1.3733(18)$ & $\mathrm{C} 11-\mathrm{C} 12$ & $1.495(2)$ \\
\hline $\mathrm{O} 3-\mathrm{C} 10$ & $1.3836(19)$ & $\mathrm{C} 12-\mathrm{C} 14$ & $1.521(2)$ \\
\hline $\mathrm{N} 1-\mathrm{C} 10$ & $1.355(2)$ & $\mathrm{C} 12-\mathrm{C} 15$ & $1.528(2)$ \\
\hline $\mathrm{N} 1-\mathrm{N} 2$ & $1.4022(17)$ & $\mathrm{C} 12-\mathrm{C} 13$ & $1.534(2)$ \\
\hline $\mathrm{N} 1-\mathrm{C} 8$ & $1.4228(19)$ & $\mathrm{C} 13-\mathrm{H} 13 \mathrm{~A}$ & 0.9800 \\
\hline $\mathrm{N} 2-\mathrm{C} 11$ & $1.284(2)$ & $\mathrm{C} 13-\mathrm{H} 13 \mathrm{~B}$ & 0.9800 \\
\hline $\mathrm{C} 1-\mathrm{C} 2$ & $1.179(3)$ & $\mathrm{C} 13-\mathrm{H} 13 \mathrm{C}$ & 0.9800 \\
\hline $\mathrm{C} 1-\mathrm{H} 1$ & 0.9500 & $\mathrm{C} 14-\mathrm{H} 14 \mathrm{~A}$ & 0.9800 \\
\hline $\mathrm{C} 2-\mathrm{C} 3$ & $1.464(3)$ & $\mathrm{C} 14-\mathrm{H} 14 \mathrm{~B}$ & 0.9800 \\
\hline $\mathrm{C} 3-\mathrm{H} 3 \mathrm{~A}$ & 0.9900 & $\mathrm{C} 14-\mathrm{H} 14 \mathrm{C}$ & 0.9800 \\
\hline $\mathrm{C} 3-\mathrm{H} 3 \mathrm{~B}$ & 0.9900 & $\mathrm{C} 15-\mathrm{H} 15 \mathrm{~A}$ & 0.9800 \\
\hline $\mathrm{C} 4-\mathrm{C} 9$ & $1.387(2)$ & C15-H15B & 0.9800 \\
\hline $\mathrm{C} 4-\mathrm{C} 5$ & $1.396(2)$ & $\mathrm{C} 15-\mathrm{H} 15 \mathrm{C}$ & 0.9800 \\
\hline $\mathrm{C} 5-\mathrm{C} 6$ & $1.373(2)$ & & \\
\hline $\mathrm{C} 4-\mathrm{O} 1-\mathrm{C} 3$ & $117.74(12)$ & $\mathrm{C} 8-\mathrm{C} 9-\mathrm{H} 9$ & 120.0 \\
\hline $\mathrm{C} 11-\mathrm{O} 3-\mathrm{C} 10$ & $106.45(12)$ & $\mathrm{O} 2-\mathrm{C} 10-\mathrm{N} 1$ & $131.27(15)$ \\
\hline $\mathrm{C} 10-\mathrm{N} 1-\mathrm{N} 2$ & $111.83(12)$ & $\mathrm{O} 2-\mathrm{C} 10-\mathrm{O} 3$ & $124.23(15)$ \\
\hline $\mathrm{C} 10-\mathrm{N} 1-\mathrm{C} 8$ & $126.62(13)$ & $\mathrm{N} 1-\mathrm{C} 10-\mathrm{O} 3$ & $104.49(13)$ \\
\hline $\mathrm{N} 2-\mathrm{N} 1-\mathrm{C} 8$ & $121.56(12)$ & $\mathrm{N} 2-\mathrm{C} 11-\mathrm{O} 3$ & $113.59(13)$ \\
\hline $\mathrm{C} 11-\mathrm{N} 2-\mathrm{N} 1$ & $103.60(12)$ & $\mathrm{N} 2-\mathrm{C} 11-\mathrm{C} 12$ & $128.70(14)$ \\
\hline $\mathrm{C} 2-\mathrm{C} 1-\mathrm{H} 1$ & 180.0 & $\mathrm{O} 3-\mathrm{C} 11-\mathrm{C} 12$ & $117.71(13)$ \\
\hline $\mathrm{C} 1-\mathrm{C} 2-\mathrm{C} 3$ & $177.0(2)$ & $\mathrm{C} 11-\mathrm{C} 12-\mathrm{C} 14$ & $108.73(13)$ \\
\hline $\mathrm{O} 1-\mathrm{C} 3-\mathrm{C} 2$ & $111.27(14)$ & $\mathrm{C} 11-\mathrm{C} 12-\mathrm{C} 15$ & $108.86(14)$ \\
\hline $\mathrm{O} 1-\mathrm{C} 3-\mathrm{H} 3 \mathrm{~A}$ & 109.4 & $\mathrm{C} 14-\mathrm{C} 12-\mathrm{C} 15$ & $110.28(16)$ \\
\hline $\mathrm{C} 2-\mathrm{C} 3-\mathrm{H} 3 \mathrm{~A}$ & 109.4 & $\mathrm{C} 11-\mathrm{C} 12-\mathrm{C} 13$ & $108.54(14)$ \\
\hline $\mathrm{O} 1-\mathrm{C} 3-\mathrm{H} 3 \mathrm{~B}$ & 109.4 & $\mathrm{C} 14-\mathrm{C} 12-\mathrm{C} 13$ & $110.56(16)$ \\
\hline $\mathrm{C} 2-\mathrm{C} 3-\mathrm{H} 3 \mathrm{~B}$ & 109.4 & $\mathrm{C} 15-\mathrm{C} 12-\mathrm{C} 13$ & $109.82(15)$ \\
\hline $\mathrm{H} 3 \mathrm{~A}-\mathrm{C} 3-\mathrm{H} 3 \mathrm{~B}$ & 108.0 & $\mathrm{C} 12-\mathrm{C} 13-\mathrm{H} 13 \mathrm{~A}$ & 109.5 \\
\hline $\mathrm{O} 1-\mathrm{C} 4-\mathrm{C} 9$ & $125.65(14)$ & $\mathrm{C} 12-\mathrm{C} 13-\mathrm{H} 13 \mathrm{~B}$ & 109.5 \\
\hline $\mathrm{O} 1-\mathrm{C} 4-\mathrm{C} 5$ & $115.88(14)$ & $\mathrm{H} 13 \mathrm{~A}-\mathrm{C} 13-\mathrm{H} 13 \mathrm{~B}$ & 109.5 \\
\hline $\mathrm{C} 9-\mathrm{C} 4-\mathrm{C} 5$ & $118.47(14)$ & $\mathrm{C} 12-\mathrm{C} 13-\mathrm{H} 13 \mathrm{C}$ & 109.5 \\
\hline $\mathrm{C} 6-\mathrm{C} 5-\mathrm{C} 4$ & $121.82(15)$ & $\mathrm{H} 13 \mathrm{~A}-\mathrm{C} 13-\mathrm{H} 13 \mathrm{C}$ & 109.5 \\
\hline $\mathrm{C} 6-\mathrm{C} 5-\mathrm{Cl} 1$ & $119.08(12)$ & $\mathrm{H} 13 \mathrm{~B}-\mathrm{C} 13-\mathrm{H} 13 \mathrm{C}$ & 109.5 \\
\hline $\mathrm{C} 4-\mathrm{C} 5-\mathrm{C} 11$ & $119.08(12)$ & $\mathrm{C} 12-\mathrm{C} 14-\mathrm{H} 14 \mathrm{~A}$ & 109.5 \\
\hline $\mathrm{C} 5-\mathrm{C} 6-\mathrm{C} 7$ & $119.30(15)$ & $\mathrm{C} 12-\mathrm{C} 14-\mathrm{H} 14 \mathrm{~B}$ & 109.5 \\
\hline $\mathrm{C} 5-\mathrm{C} 6-\mathrm{H} 6$ & 120.4 & $\mathrm{H} 14 \mathrm{~A}-\mathrm{C} 14-\mathrm{H} 14 \mathrm{~B}$ & 109.5 \\
\hline
\end{tabular}




\begin{tabular}{|c|c|c|c|}
\hline $\mathrm{C} 7-\mathrm{C} 6-\mathrm{H} 6$ & 120.4 & $\mathrm{C} 12-\mathrm{C} 14-\mathrm{H} 14 \mathrm{C}$ & 109.5 \\
\hline $\mathrm{C} 8-\mathrm{C} 7-\mathrm{C} 6$ & $119.77(14)$ & $\mathrm{H} 14 \mathrm{~A}-\mathrm{C} 14-\mathrm{H} 14 \mathrm{C}$ & 109.5 \\
\hline $\mathrm{C} 8-\mathrm{C} 7-\mathrm{Cl} 2$ & $121.50(12)$ & $\mathrm{H} 14 \mathrm{~B}-\mathrm{C} 14-\mathrm{H} 14 \mathrm{C}$ & 109.5 \\
\hline $\mathrm{C} 6-\mathrm{C} 7-\mathrm{Cl} 2$ & $118.73(12)$ & $\mathrm{C} 12-\mathrm{C} 15-\mathrm{H} 15 \mathrm{~A}$ & 109.5 \\
\hline $\mathrm{C} 7-\mathrm{C} 8-\mathrm{C} 9$ & $120.57(14)$ & $\mathrm{C} 12-\mathrm{C} 15-\mathrm{H} 15 \mathrm{~B}$ & 109.5 \\
\hline $\mathrm{C} 7-\mathrm{C} 8-\mathrm{N} 1$ & $120.52(14)$ & $\mathrm{H} 15 \mathrm{~A}-\mathrm{C} 15-\mathrm{H} 15 \mathrm{~B}$ & 109.5 \\
\hline $\mathrm{C} 9-\mathrm{C} 8-\mathrm{N} 1$ & $118.89(14)$ & $\mathrm{C} 12-\mathrm{C} 15-\mathrm{H} 15 \mathrm{C}$ & 109.5 \\
\hline $\mathrm{C} 4-\mathrm{C} 9-\mathrm{C} 8$ & $120.03(14)$ & $\mathrm{H} 15 \mathrm{~A}-\mathrm{C} 15-\mathrm{H} 15 \mathrm{C}$ & 109.5 \\
\hline $\mathrm{C} 4-\mathrm{C} 9-\mathrm{H} 9$ & 120.0 & $\mathrm{H} 15 \mathrm{~B}-\mathrm{C} 15-\mathrm{H} 15 \mathrm{C}$ & 109.5 \\
\hline $\mathrm{C} 10-\mathrm{N} 1-\mathrm{N} 2-\mathrm{C} 11$ & $-1.55(17)$ & $\mathrm{O} 1-\mathrm{C} 4-\mathrm{C} 9-\mathrm{C} 8$ & $179.41(15)$ \\
\hline $\mathrm{C} 8-\mathrm{N} 1-\mathrm{N} 2-\mathrm{C} 11$ & $178.49(14)$ & $\mathrm{C} 5-\mathrm{C} 4-\mathrm{C} 9-\mathrm{C} 8$ & $-1.3(2)$ \\
\hline $\mathrm{C} 4-\mathrm{O} 1-\mathrm{C} 3-\mathrm{C} 2$ & $70.42(18)$ & $\mathrm{C} 7-\mathrm{C} 8-\mathrm{C} 9-\mathrm{C} 4$ & $-0.4(2)$ \\
\hline $\mathrm{C} 3-\mathrm{O} 1-\mathrm{C} 4-\mathrm{C} 9$ & $5.2(2)$ & $\mathrm{N} 1-\mathrm{C} 8-\mathrm{C} 9-\mathrm{C} 4$ & $177.85(14)$ \\
\hline $\mathrm{C} 3-\mathrm{O} 1-\mathrm{C} 4-\mathrm{C} 5$ & $-174.11(14)$ & $\mathrm{N} 2-\mathrm{N} 1-\mathrm{C} 10-\mathrm{O} 2$ & $-176.02(18)$ \\
\hline $\mathrm{O} 1-\mathrm{C} 4-\mathrm{C} 5-\mathrm{C} 6$ & $-178.05(14)$ & $\mathrm{C} 8-\mathrm{N} 1-\mathrm{C} 10-\mathrm{O} 2$ & $3.9(3)$ \\
\hline $\mathrm{C} 9-\mathrm{C} 4-\mathrm{C} 5-\mathrm{C} 6$ & $2.6(2)$ & $\mathrm{N} 2-\mathrm{N} 1-\mathrm{C} 10-\mathrm{O} 3$ & $2.34(17)$ \\
\hline $\mathrm{O} 1-\mathrm{C} 4-\mathrm{C} 5-\mathrm{Cl1}$ & $3.8(2)$ & $\mathrm{C} 8-\mathrm{N} 1-\mathrm{C} 10-\mathrm{O} 3$ & $-177.70(14)$ \\
\hline $\mathrm{C} 9-\mathrm{C} 4-\mathrm{C} 5-\mathrm{Cl} 1$ & $-175.58(12)$ & $\mathrm{C} 11-\mathrm{O} 3-\mathrm{C} 10-\mathrm{O} 2$ & $176.34(16)$ \\
\hline $\mathrm{C} 4-\mathrm{C} 5-\mathrm{C} 6-\mathrm{C} 7$ & $-2.1(2)$ & $\mathrm{C} 11-\mathrm{O} 3-\mathrm{C} 10-\mathrm{N} 1$ & $-2.17(16)$ \\
\hline $\mathrm{C} 11-\mathrm{C} 5-\mathrm{C} 6-\mathrm{C} 7$ & $176.10(12)$ & $\mathrm{N} 1-\mathrm{N} 2-\mathrm{C} 11-\mathrm{O} 3$ & $0.07(17)$ \\
\hline $\mathrm{C} 5-\mathrm{C} 6-\mathrm{C} 7-\mathrm{C} 8$ & $0.3(2)$ & $\mathrm{N} 1-\mathrm{N} 2-\mathrm{C} 11-\mathrm{C} 12$ & $179.98(15)$ \\
\hline $\mathrm{C} 5-\mathrm{C} 6-\mathrm{C} 7-\mathrm{Cl} 2$ & $-179.26(12)$ & $\mathrm{C} 10-\mathrm{O} 3-\mathrm{C} 11-\mathrm{N} 2$ & $1.36(18)$ \\
\hline $\mathrm{C} 6-\mathrm{C} 7-\mathrm{C} 8-\mathrm{C} 9$ & $1.0(2)$ & $\mathrm{C} 10-\mathrm{O} 3-\mathrm{C} 11-\mathrm{C} 12$ & $-178.56(14)$ \\
\hline $\mathrm{Cl} 2-\mathrm{C} 7-\mathrm{C} 8-\mathrm{C} 9$ & $-179.51(12)$ & $\mathrm{N} 2-\mathrm{C} 11-\mathrm{C} 12-\mathrm{C} 14$ & $-2.4(2)$ \\
\hline $\mathrm{C} 6-\mathrm{C} 7-\mathrm{C} 8-\mathrm{N} 1$ & $-177.28(14)$ & $\mathrm{O} 3-\mathrm{C} 11-\mathrm{C} 12-\mathrm{C} 14$ & $177.51(15)$ \\
\hline $\mathrm{Cl} 2-\mathrm{C} 7-\mathrm{C} 8-\mathrm{N} 1$ & $2.2(2)$ & $\mathrm{N} 2-\mathrm{C} 11-\mathrm{C} 12-\mathrm{C} 15$ & $-122.58(19)$ \\
\hline $\mathrm{C} 10-\mathrm{N} 1-\mathrm{C} 8-\mathrm{C} 7$ & $65.3(2)$ & $\mathrm{O} 3-\mathrm{C} 11-\mathrm{C} 12-\mathrm{C} 15$ & $57.33(19)$ \\
\hline $\mathrm{N} 2-\mathrm{N} 1-\mathrm{C} 8-\mathrm{C} 7$ & $-114.74(16)$ & $\mathrm{N} 2-\mathrm{C} 11-\mathrm{C} 12-\mathrm{C} 13$ & $117.92(18)$ \\
\hline $\mathrm{C} 10-\mathrm{N} 1-\mathrm{C} 8-\mathrm{C} 9$ & $-112.99(18)$ & $\mathrm{O} 3-\mathrm{C} 11-\mathrm{C} 12-\mathrm{C} 13$ & $-62.17(19)$ \\
\hline $\mathrm{N} 2-\mathrm{N} 1-\mathrm{C} 8-\mathrm{C} 9$ & $66.97(19)$ & & \\
\hline
\end{tabular}

Hydrogen-bond geometry $\left(A,{ }^{\circ}\right)$

$\mathrm{Cg} 1$ and $\mathrm{Cg} 2$ are the centroids of the $\mathrm{O} 3 / \mathrm{C} 10 / \mathrm{N} 1 / \mathrm{N} 2 / \mathrm{C} 11$ and $\mathrm{C} 4-\mathrm{C} 9$ rings, respectively.

\begin{tabular}{lllll}
\hline$D-\mathrm{H} \cdots A$ & $D-\mathrm{H}$ & $\mathrm{H} \cdots A$ & $D \cdots A$ & $D-\mathrm{H}^{\cdots} A$ \\
\hline $\mathrm{C} 1-\mathrm{H} 1 \cdots \mathrm{O} 2^{\mathrm{i}}$ & 0.95 & 2.47 & $3.401(3)$ & 168 \\
$\mathrm{C} 13-\mathrm{H} 13 B^{\cdots} \cdots \mathrm{O} 2^{\mathrm{ii}}$ & 0.98 & 2.51 & $3.429(2)$ & 155 \\
$\mathrm{C} 3-\mathrm{H} 3 B \cdots \mathrm{N} 2^{\mathrm{iii}}$ & 0.99 & 2.64 & $3.607(2)$ & 166 \\
$\mathrm{C} 13-\mathrm{H} 13 A \cdots \mathrm{C} 1^{\mathrm{iv}}$ & 0.98 & 2.85 & $3.811(2)$ & 168 \\
$\mathrm{C} 14-\mathrm{H} 14 \mathrm{~b} \cdots C g 2^{\mathrm{ii}}$ & 0.98 & 2.99 & $3.396(2)$ & 106 \\
$\mathrm{C} 15-\mathrm{H} 15 \mathrm{c} \cdots C g 1^{\mathrm{v}}$ & 0.98 & 2.80 & $3.497(2)$ & 129
\end{tabular}

Symmetry codes: (i) $-x,-y+2,-z$; (ii) $x, y, z+1$; (iii) $x,-y+3 / 2, z-1 / 2$; (iv) $-x+1,-y+2,-z+1$; (v) $-x,-y+2,-z+2$. 